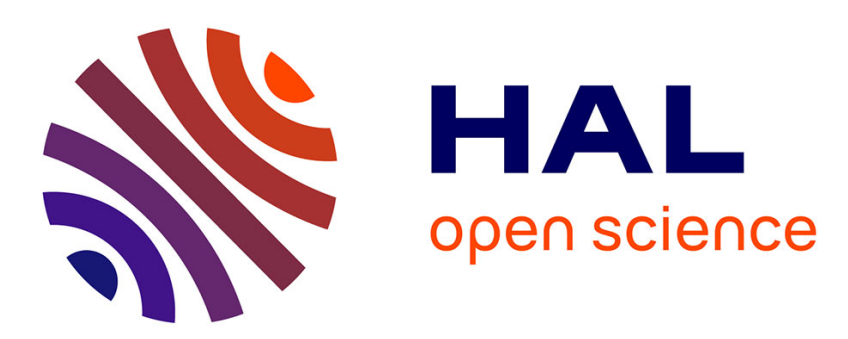

\title{
Recent results on the stability of anisotropic stellar systems
}

Jérôme Perez, Jean-Jacques Aly

\section{To cite this version:}

Jérôme Perez, Jean-Jacques Aly. Recent results on the stability of anisotropic stellar systems. Ergodic Concepts in Stellar Dynamics, D. Pfenniger, Mar 2003, Geneva, Switzerland. hal-01141508

\section{HAL Id: hal-01141508 \\ https://hal.science/hal-01141508}

Submitted on 13 Apr 2015

HAL is a multi-disciplinary open access archive for the deposit and dissemination of scientific research documents, whether they are published or not. The documents may come from teaching and research institutions in France or abroad, or from public or private research centers.
L'archive ouverte pluridisciplinaire HAL, est destinée au dépôt et à la diffusion de documents scientifiques de niveau recherche, publiés ou non, émanant des établissements d'enseignement et de recherche français ou étrangers, des laboratoires publics ou privés. 


\title{
Recent results on the stability of anisotropic stellar systems
}

\author{
Jérôme Perez ${ }^{1,2}$ \\ Jean-Jacques Aly ${ }^{1}$ \\ 1 Service d'Astrophysique - CE Saclay - 91191 Gif sur Yvette Cédex - France \\ 2 ETCA/CREA - 16 bis av. Prieur de la côte d'or - 94114 Arcueil Cédex -France
}

\begin{abstract}
We present a new approach to deal with the problem of stability of collisionless stelar systems. This technique based on the symplectic structure of the Vlasov-Poisson system allow us to have a new stability criteria for general systems very usefull in the anisotropic spherical case.
\end{abstract}

\section{Introduction}

As it was remarked by Zwicky in the fiveties, owing to their physical aspect, galaxies and comparable stellar systems seems to be relaxed, but their collisional relaxation time is in general larger than the age of the universe. So, there exist a collisionless epoch inportant in the life of stellar systems which govern a part of their dynamical evolution. Taking into account the statistical behaviour of their components, stellar systems can be treated by a Kinetic Theory. However, as it was stessed by several authors (see Binney and Tremaine,1986- hereafter BT - for a review) classical stability techniques stops when the problem become inhomogeneous and anisotropic. In this sence, folowing the pioneer works of Bartolomew,1971 reactualized by Kandrup 1991a , we present here a new symplectic approach of dynamical evolution of collisionless stellar systems. This approach permit to obtain a quite simple and very general stability criteria, which, in the special case of spherical isopotential curves allow us to generalise an important isotropic result called sometimes Antonov-Lebovitz Theorem.

\section{A symplectic stability criterion}

As it was shown by Morrison,1982 the standard Vlasov equation can be exprimed using functionals. Indeed, if $F$ is any functionnal involving in the phase space, the motion evolution equation can be written

$$
\dot{F}[f]=\int f\left[\frac{\delta F}{\delta f}, \frac{\delta H}{\delta f}\right] d \Gamma:=\{F, H\}[f]
$$

where [,] denotes the standard Poisson bracket in the canonical conjugated variables $p$ and $q,\{$,$\} have all the properties of a Lie bracket and \delta / \delta f$ meaning a functional derivative operation . 
Owing to the fact that any physical perturbation of any initial state $f_{o}$ can be described by a generator $g$ which is an hamiltonian-like function representing the canonical transformation effectued, (e.g. Bartolomew 1971), we can find a general Taylor expand for any functional $F$ during this perturbation

$$
\begin{aligned}
\hat{F}\left[f_{o}\right] & =F\left[f_{0}\right]-\{G, F\}+\frac{1}{2}\{G,\{G, F\}\}-\frac{1}{3 !}\{G,\{G,\{G, F\}\}\}+\cdots \\
& =\left(e^{\{\cdot, G\}} F\right)\left[f_{o}\right]=F\left[e^{[g, \cdot]} f_{o}\right]
\end{aligned}
$$

where $G$ is such that $\delta G / \delta f=g$ is the Functional generator.

It is important to note that development (2) is true for any functional, so we can apply it to all functional intervening in our problem as energy, entropy or more complicated one.

Applying (2) in the special case of the total energy of the system

$$
H[f]=\int d \Gamma \frac{p^{2}}{2 m} f(q, p, t)-\frac{G m^{2}}{2} \int d \Gamma \int d \Gamma^{\prime} \frac{f(q, p, t) f\left(q^{\prime}, p^{\prime}, t\right)}{\left|q-q^{\prime}\right|}
$$

, and choosing for $f_{o}$ a steady state, the first order energy variation is clearly vanishing, and the second order can be written

$$
H^{(2)}\left[f_{o}\right]=-\frac{1}{2} \int[g, E]\left[g, f_{o}\right] d \Gamma-\frac{G m^{2}}{2} \int d \Gamma \int d \Gamma^{\prime} \frac{\left[g, f_{o}\right] \cdot\left[g^{\prime}, f_{o}^{\prime}\right]}{\left|q-q^{\prime}\right|}
$$

where $E$ is the single-particle energy, functional derivative of $H[f]$. The stability of the system against some perturbations generated by some $g$, can now be investigated in the anisotropic inhomogeneous case by the study of the sign of $H^{(2)}\left[f_{o}\right]$. However, this development give to us only a sufficient condition for stability. Indeed, we cannot make, as (Laval,Mercier,Pellat,1965), a connection between $H^{(2)}\left[f_{o}\right]$ and some definite inner product to have a general energy principle. As it is quoted in Larson,1991 the inner-product related to our general problem is indefinite, and in this case relations like Schwartz's inequality fall and not allow us to connect our energy variation to some dynamical variable to diagnostic linear instabilities. However, as it is illustred in Kandrup,1991b negativeness of $H^{(2)}\left[f_{o}\right]$ can imply secular instability, and a way is open in this weak version of instability.

\section{Stability Results}

As it is quoted in BT, stability problem of a collisionless stellar system and of a gaz volume in gravitational interaction are closely related. In fact, in many cases, the hydrodynamic problem (when it is identifiable) is simpler than it stellar analog. Indeed, hydrodynamic problem is a three dimensional one when stellar systems have six degree of freedom. Hence a technique to simplify the study of stellar systems consist to average over velocities, when it is possible, to deal with the hydrodynamic counterpart of the problem . One 
of the most important isotropic result, sometimes called Antonov-Lebovitz Theorem(ALT) (see BT), is built on this technique and assure the stability of $f_{o}(E)$ 's systems against non-radial perturbations.

In a recent paper (Aly and Perez, 1992), we present a new demonstration of this important result. This is the combination of this new method and the symplectic approach of the stability criterion which allow us to obtain our result on the stability of spherical stellar systems.

Before to give the proof with have to install the context and define the class of perturbations which allow us to use the technique.

A stationary spherical system has a distribution function which depends only on the energy $E$ and the squared norm $L^{2}$ of the angular momentum $L$. $f_{o}=f_{o}\left(E, L^{2}\right)$. We assume here that

$$
f_{E}:=\frac{\partial f_{0}}{\partial E} \leq 0 \text { and } f_{L^{2}}:=\frac{\partial f_{0}}{\partial L^{2}} \leq 0
$$

We want to consider the stability of such an equilibrium with respect to the class of preserving perturbations, which are generated by all the functions $g$ satisfying $\left[g, L^{2}\right]=0$. It is easy to see what is preciselly the class of preserving perturbation writing the definition of a perturbation g-generated

$$
f_{1}=\left[g, f_{o}\left(E, L^{2}\right)\right]=f_{E}[g, E]+f_{L^{2}}\left[g, L^{2}\right]
$$

This equation is written in the general anisotropic spherical case. In order to clarify the situation, it is clear that in the isotropic case where $f_{o}$ depends only of $E$ all perturbations are preserving because $g$ always commutes with $L^{2}$.Moreover, the stability of isotropic systems in the (5) conditions is well known. On the other hand, in the case of purelly radial orbit system, with distribution function of the form $f_{o}\left(E, L^{2}\right)=\phi(E) . \delta\left(L^{2}\right)$ (e.g. Fridman and Polyachenko,1984), where $\phi$ is any smooth function and $\delta$ denotes the dirac distribution, one can show that there no exists non vanishing $g$ such that $\left[g, L^{2}\right]=0$ and $f_{1} \neq 0$ simultaneously. In this sence we say that purely radial orbit systems cannot receive preserving perturbations.

In the intermediate case preserving perturbations are generated by all functions of the $g\left(L^{2}, L_{x}, L_{y}, L_{z}\right)$ form, for example all radial perturbation is also preserving. Non radial and preserving perturbations are clearly mathematically defined and numerically seen as not forming a vanishing set, but we haven't seen their physical interpretation.

In the general spherical case one can split the perturbation $f_{1}$ in two parts with one invariant under rotation

$$
\begin{gathered}
f_{1}=\overline{f_{1}}+\delta f_{1} \\
\overline{f_{1}}(q, p)=\int f_{1}(R(q), R(p)) d R \text { and } \int \delta f_{1}(R(q), R(p)) d R=0
\end{gathered}
$$

where the averaging is made other all possibles rotations $R$. Hence, one can show that the second order variation of the energy splits in $H^{(2)}\left[f_{1}\right]=$ $H^{(2)}\left[\overline{f_{1}}\right]+H^{(2)}\left[\delta f_{1}\right]$. The first part being positive by Kandrup and Sygnet, 
1985 Theorem, we study the second non-radial part and show (using ower revisited proof of ALT and the well known Wirtinger inequality)

$$
H^{(2)}\left[\delta f_{1}\right] \geq \frac{1}{2} \int \frac{\left(\delta \rho_{1}\right)^{2}}{\left|f_{E}\right|} d r-\frac{G m^{2}}{2} \int d r \int d r^{\prime} \frac{\delta \rho_{1} \delta \rho_{1}^{\prime}}{\left|q-q^{\prime}\right|} \geq 0
$$

where the non radial part of the perturbed density $\delta \rho_{1}$ is directly obtained from $\delta f_{1}$ by a velocity averaging. This result assuring the stability of any anisotropic spherical stellar system - having the properties (5) - against preserving perturbations.Therefore, it should be stressed that the preserving character is not dynamically robust. To have a greater signification this stability result must be completed by a numeric experience concerning the relation existing the nature of perturbation affecting a wide class of anisotropic spherical systems and their preserving character. These kind of simulations are under investigations and preliminary results tend to confirm that in most of cases, stables systems are preserving-perturbed and unstable systems are non-preseving perturbed.(Perez et al,1993, Perez and Alimi,1993).

\section{References}

Aly, J.J.,Perez,J., 1992, Mon. Not. Roy. Astron. Soc., 259, 95

Bartolomew,P., 1971, Mon. Not. Roy. Astron. Soc., 151, 333

Binney, J., Tremaine S.,1984, Galactic Dynamics, Princeton Univ. Press.

Fridman,A.M.,Polyachenko,V.L., 1984, Physics of gravitating systems, New York, Springer

Kandrup,H.E.,1990, Astrophys. J., 351, 104

Kandrup,H.E.,1991a, Astrophys. J., 370, 312

Kandrup,H.E.,1991b, Astrophys. J., 380, 511

Kandrup, H., Sygnet, J.F., 1985, Astrophys.J., 298, 27

Laval,G.,Mercier,C,Pellat,R.,1965, Nuclear fusion, 5, 156

Larson,J., 1991, Physics Rewiew Let., 66, 1466

Morisson,P.J., 1980, Physics Let. A, 80, 383

Perez,J.,Alimi,J.M.,Aly,J.J.,Scholl,H.,1993, Procedings of the gravitational meeting of Aussois,Springer, in press

Perez,J.,Aly,J.J., 1993, Mon. Not. Roy. Astron. Soc., in preparation

Perez,J.,Alimi,J.M.,Aly,J.J.,Scholl,H., 1993, Mon. Not. Roy. Astron. Soc., in preparation 